\title{
Analysis of Temporal Pulse Development in Passively Mode-Locked Lasers
}

\author{
F. Graf, G. Pleininger, and A. Penzkofer \\ Naturwissenschaftliche Fakultät II - Physik, Universität, D-8400 Regensburg, \\ Fed. Rep. Germany
}

Received 5 March 1984/Accepted 31 March 1984

\begin{abstract}
Pulse-shortening and pulse broadening effects in passively mode-locked lasers are analysed. A steady-state pulse duration limit is calculated and compared with round-trip simulations. The numerical calculations apply to a picosecond Nd : glass laser. Methods of short-pulse generation are discussed.
\end{abstract}

PACS: 42.55R, 42.60D, 42.65

In passively mode-locked pulsed lasers the statistical spontaneous emission bursts have a duration approximately equal to the inverse of the fluorescence linewidth. In the linear laser phase (constant dye absorption) the finite spectral gain profile of the active medium narrows the spectral width and broadens the duration of the circulating spikes. In the nonlinear laser phase (nonlinear dye transmission, mode-locking region) the most intense spike is preferentially amplified and strong background discrimination is achieved. Pulse shortening occurs in the saturable absorber cell. (For reviews on mode-locking see [1-7].) The natural mode selection in the active medium acts against the shortening. Intensity dependent loss mechanisms like two-photon absorption in $\mathrm{Nd}$ : glass rods [8] broaden the pulse duration at high laser intensities [9].

In this paper we analyse the pulse shortening and pulse broadening effects in passively mode-locked lasers. Intensity-dependent stationary pulse durations are found by equating pulse shortening and pulse broadening per round-trip in the laser oscillator. In the mode-locking region the transient temporal development of a picosecond pulse with constant background level is simulated and the approach to the stationary situation is studied.

The theory presented applies to all passively modelocked lasers, either pulsed or continuously working [10]. The numerical calculations in this paper apply to a passively mode-locked $\mathrm{Nd}$ :glass laser. The calculations reveal conditions for optimum shortpulse generation in the oscillator. Preliminary measurements with a Pockels cell intensity limiter in the resonator led to pulse shortening in agreement with the theory.

\section{Stationary Pulse Durations}

The changes of pulse duration per round-trip in the oscillator are analysed. Pulses are broadened by the finite spectral width of the gain and by intensity dependent nonlinear losses. Here pulse broadening by two-photon absorption in $\mathrm{Nd}$ : glass rods is discussed. Pulse shortening occurs in the nonlinear transmission region of the saturable absorber.

The pulse duration ratio $\beta=\Delta t_{L, 0} / \Delta t_{L, i}$, where $\Delta t_{L, 0}$ is the pulse duration after $n+1$ round-trips and $\Delta t_{L, i}$ the pulse duration after $n$ round-trips, is given by

$\beta=\beta_{\mathrm{GAIN}} \beta_{\mathrm{TPA}} \beta_{\mathrm{DYE}}$.

$\beta_{\mathrm{GAIN}}$ is the ratio of pulse broadening by the finite spectral width of the gain profile. $\beta_{\text {TPA }}$ represents the ratio of pulse broadening by two-photon absorption in the active medium. $\beta_{\mathrm{DYE}}$ is the pulse shortening ratio of the saturable dye.

The spectral gain profile of the active medium approximately has the form [9]

$g\left(v-v_{0}\right)=G(v) / G\left(v_{0}\right)$ 


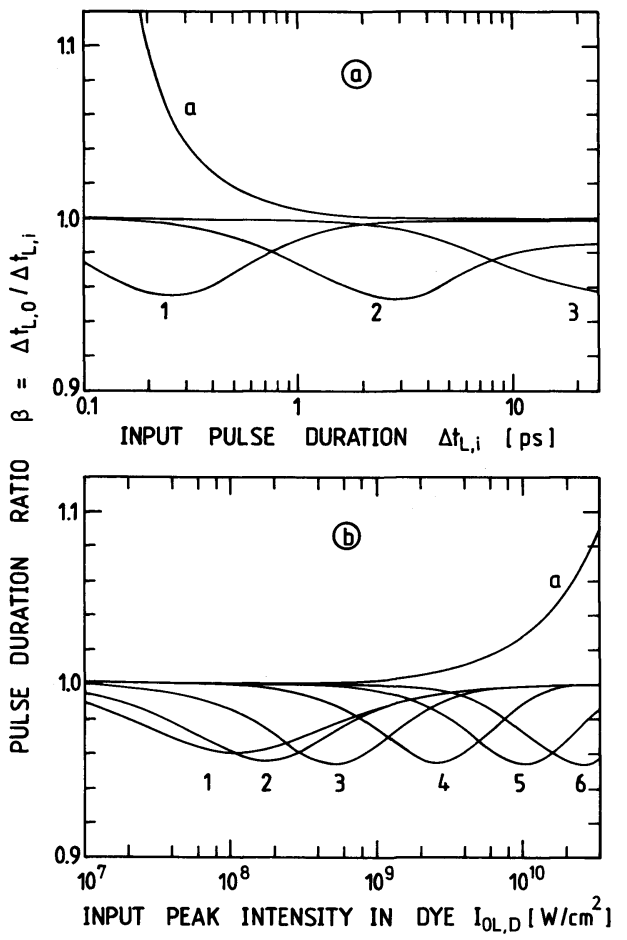

Fig. 1a and b. Pulse duration changes per round-trip in the oscillator. Curves are calculated with parameters of Table 1. (a) Curve $a$, pulse broadening due to finite spectral gain width $\beta_{\text {GAIN }}$. Curves $1-3$, pulse shortening in saturable dye $\beta_{\mathrm{DYE}}$ for (1) $I_{O L, D}=10^{10} \mathrm{~W} / \mathrm{cm}^{2}$, (2) $10^{9} \mathrm{~W} / \mathrm{cm}^{2}$, and (3) $10^{8} \mathrm{~W} / \mathrm{cm}^{2}$. (b) Curve $a$, pulse broadening due to two-photon absorption $\beta_{\text {TPA }}$. Curves $1-6$, pulse shortening in dye $\beta_{\mathrm{DYE}}$ for (1) $\Delta t_{L, i}=500 \mathrm{ps}$ (steady state), (2) $20 \mathrm{ps,} \mathrm{(3)} 5 \mathrm{ps,} \mathrm{(4)} 1 \mathrm{ps}$, (5) $0.25 \mathrm{ps,} \mathrm{and}$ (6) $0.1 \mathrm{ps}$

with the gain factors

$G(v)=\exp \left\{2 l_{A} \sigma_{A} N_{i} \exp \left[-\left(v-v_{0}\right)^{2} / v_{G}^{2}\right]\right\}$

and

$G\left(v_{0}\right)=\exp \left(2 l_{A} \sigma_{A} N_{i}\right) \simeq \frac{G_{N}}{R_{1} R_{2} T_{l} T_{0}^{2}} ;$

$l_{A}$ is the length of the active medium, $\sigma_{A}$ is the stimulated emission cross-section. $N_{i}=N_{2, A}-N_{1, A}$ represents the number density of inverted active ions that emit at frequency $v_{0}$. A Gaussian spectral emission shape is assumed with an $1 / \mathrm{e}$ width of $v_{G}$. $G_{N}$ is the net gain per round-trip at the end of the linear phase. $R_{1}$ and $R_{2}$ are the mirror reflectivities. $T_{l}$ accounts for linear losses and $T_{0}$ is the small signal single-pass transmission of the saturable dye. The full halfwidth of the gain profile $g\left(v-v_{0}\right)$ is $\Delta v_{g}$ [found by setting $\left.g\left(v-v_{0}\right)=1 / 2\right]$. The temporal response function corresponding to the spectral gain profile $g\left(v-v_{0}\right)$ is approximately of Gaussian shape and has a full halfwidth of $\Delta t_{g}=0.44 / \Delta v_{g}$. The temporal pulse broadening per round-trip is given by the convolution
Table 1. Parameters used in calculations

$\begin{array}{ll}\text { Resonator } & \\ \text { Configuration: } & \text { hemi-confocal } \\ \text { Mirror reflectivities: } & R_{1}=0.3, R_{2}=0.997 \\ \text { Linear transmission: } & T_{l}=0.9 \\ \text { Focusing ratio: } & \kappa=I_{O L, D} / I_{O L, A}=6.5\end{array}$

\section{Active Medium}

Type: Nd-phosphate glass LG 703 from Glaswerke Schott Fluorescence halfwidth $\Delta v_{G}=189 \mathrm{~cm}^{-1}$ (FWHM) Spectral gain width $\quad \Delta v_{g}=163 \mathrm{~cm}^{-1}$ (FWHM) [9] Pumped length $\quad l_{A}=10 \mathrm{~cm}$ Rod length $\quad l_{R}=13 \mathrm{~cm}$

Stimulated emission cross-section $\sigma_{A}=4.1 \times 10^{-20} \mathrm{~cm}^{2}$ Two-photon absorption coefficient $\alpha^{(2)}=4 \times 10^{-12} \mathrm{~cm} / \mathrm{W}$

\section{Saturable Absorber}

Type: Kodak No. 9860

Absorption recovery time $\tau_{D}=7 \mathrm{ps}[16]$

Absorption cross-section $\sigma_{D}=3.7 \times 10^{-16} \mathrm{~cm}^{2}$

Excited state absorption cross-section $\sigma_{\mathrm{ex}}=0$ (assumed)

Single pass small signal transmission $T_{0}=0.85$

\section{Stationary Conditions}

Pulse shape: Gaussian

Transient Start Conditions (End of Linear Phase)

Pulse shape $s(t)=(1-\beta) \exp \left(-t^{2} / t_{0}^{2}\right)+\beta$

Duration $\Delta t=t_{0} /\left[2(\ln 2)^{1 / 2}\right]=9 \mathrm{ps}, t_{0}=5.4 \mathrm{ps}$

Background to peak intensity ratio $\beta=I_{B} / I_{0 L, 1}=1 / 7$

Peak intensity $I_{0 L, D, 1}=10^{6} \mathrm{~W} / \mathrm{cm}^{2}$

Net gain $G_{N}=1.03$

Saturation factor $\delta=0.98$

of the pulse shape with the response function. In our calculations the broadening ratio is approximated by

$\beta_{\mathrm{GAIN}}=\frac{\left(\Delta t_{L, i}^{2}+\Delta t_{g}^{2}\right)^{1 / 2}}{\Delta t_{L, i}}$.

In Fig. 1a the curve $a$ shows the dependence of $\beta_{\text {GAIN }}$ on $\Delta t_{L, i}$ for a Nd-phosphate glass oscillator with the laser parameters listed in Table 1. The steep rise of $\beta_{\text {GAIN }}$ - when $\Delta t_{L, i}$ approaches $\Delta v_{g}^{-1}$ sets a lower limit to the obtainable pulse duration. The halfwidth of the gain profile $\Delta v_{g}$ is slightly smaller than the halfwidth of the fluorescence profile $\Delta v_{G}=2[\ln (2)]^{1 / 2} v_{G}$. A reasonable estimate of $\beta_{\text {GAIN }}$ is already obtained by using $\Delta v_{G}$ instead of $\Delta v_{g}$.

Intensity dependent losses broaden bell-shaped light pulses. Two-photon absorption is observed in $\mathrm{Nd}$ :glass lasers [8]. The two-photon intensity transmission per round-trip is

$T_{\mathrm{TPA}}=\frac{1}{1+\alpha^{(2)} 2 l_{R} I_{0 L, A, i} \exp \left(-t^{2} / t_{0}^{2}\right)} ;$ 
$\alpha^{(2)}$ is the two-photon absorption cross-section. $l_{R}$ is the laser rod length and $I_{0 L, A, i}$ is the pulse peak intensity in the laser rod. $t_{0}=\Delta t_{L, i} /\left[2(\ln 2)^{1 / 2}\right]$ is the $1 / \mathrm{e}$ temporal pulse width. A Gaussian temporal pulse shape is assumed. The spatially averaged two-photon power transmission $\bar{T}_{\text {TPA }}$ is found approximately by using an average intensity $\bar{I}_{O L, A, i}=0.5 \times I_{0 L, A, i}$. The temporal broadening $\beta_{\text {TPA }}$ is found by calculating the halfwidth $\Delta t_{L, 0}$ of the transmitted pulse of intensity distribution $I_{L, A, 0}(t)=\bar{T}_{\mathrm{TPA}} I_{L, A, i}(t)$ and forming the ratio $\beta_{\mathrm{TPA}}=\Delta t_{L, 0} / \Delta t_{L, i}$. The result is

$\beta_{\mathrm{TPA}}=\frac{\left[\ln 2+\ln \left(1+0.5 \alpha^{(2)} l_{R} I_{0 L, A, i}\right)\right]^{1 / 2}}{(\ln 2)^{1 / 2}}$.

The curve $a$ of Fig. $1 \mathrm{~b}$ represents the dependence of the broadening factor $\beta_{\text {TPA }}$ on input peak intensity $I_{O L, D}$ in the saturable absorber for the parameters listed in Table 1. A ratio of $\kappa=I_{0 L, D} / I_{0 L, A}=6.5$ is used in the calculations. The ratio applies to a hemiconfocal resonator with contacted dye cell at the plane mirror (used in the experiments) [10]. With increasing pulse intensity the temporal pulse broadening due to two-photon absorption rises strongly. The pulse broadening depends on the temporal pulse shape. Only rectangularly shaped pulses $\left(I_{L}(t)\right.$ $\left.=I_{0 L}\left[\theta(t)-\theta\left(t-\Delta t_{L}\right)\right] ; \theta(\zeta<0)=0, \theta(\zeta>0)=1\right)$ are not broadened by two-photon absorption (constant intensity causes constant loss).

The pulse shortening in the saturable dye is described here by a special model of isotropic interaction of light with a four-level system: The laser light excites dye molecules from the $S_{0}$-ground state (1) to a FranckCondon level in the $S_{1}$ state (2). From there the molecules relax infinitely fast to a temporal equilibrium position (3) in the $S_{1}$ state from where the molecules decay to the ground state (1). Excited state absorption from level (3) to a higher lying state (4) with infinitely fast relaxation is included. This model leads to the equation system

$\frac{\partial N_{3, D}}{\partial t}=\frac{I_{L, D}(t)}{h v_{L}} \sigma_{D}\left(N_{0, D}-N_{3, D}\right)-\frac{N_{3, D}}{\tau_{D}}$,

$\frac{\partial I_{L}}{\partial z}=-I_{L} \sigma_{D}\left(N_{0, D}-N_{3, D}\right)-I_{L} \sigma_{\mathrm{ex}} N_{3, D}$.

$N_{0, D}=N_{1, D}+N_{3, D}$ is the total number density of dye molecules. $\sigma_{D}$ is the ground-state absorption crosssection. $\sigma_{\mathrm{ex}}$ denotes the excited state absorption. $\tau_{D}$ is the absorption recovery time. The initial conditions are $N_{3, D}(t=-\infty, z)=0$ and $I_{L, D}(t, z=0)$ $=I_{0 L, D} \exp \left(-t^{2} / t_{0}^{2}\right)$. The isotropic calculations for spatial rectangular pulses, (8) and (9), give about the same result for energy transmission and pulse shortening as anisotropic calculations for spatial Gaussian pulses.
The shortening of Gaussian light pulses by passing through the contacted saturable absorber cell is depicted in Fig. 1a and b. The parameters used in the calculations belong to the mode-locking dye Kodak No. 9860 (Table 1). The Curves 1-3 in Fig. 1a present $\beta_{\mathrm{DYE}}$ versus $\Delta t_{L, i}$ for fixed values of $I_{0 L, D}$ while the Curves $1-6$ in Fig. $1 \mathrm{~b}$ belong to $\beta_{\mathrm{DYE}}\left(I_{0 L, D}\right)$ for various input pulse durations $\Delta t_{L, i}$.

The saturable absorber has an optimum intensity region of efficient pulse shortening. This optimum intensity region starts approximately at the saturation intensity $I_{S}=h v_{L} /\left(\sigma_{D} \tau_{D}\right)$ for pulses of long duration (steady state situation) and shifts to higher intensity values with decreasing input pulse duration. The minimum shortening ratio $\beta_{\mathrm{DYE}, \min }$ is nearly independent of the ratio $\tau_{D} / \Delta t_{L, i}$. The pulse shortening ratio is increased with decreasing initial dye transmission $T_{0}$. Excited state absorption reduces the pulse shortening effect somewhat. The pulse shortening ratio is also dependent on the pulse shape [11].

More refined models of saturable absorption are described in $[12,13]$. The pulse shortening behaviour of the more complex models is the same. Additional pulse shortening by transient gratings formed in the contacted dye cell $[14,15]$ is not included in our analysis.

A stationary pulse duration is achieved if the pulse broadening effects are exactly compensated by the pulse shortening effects, i.e. $\beta=\beta_{\mathrm{GAIN}} \beta_{\mathrm{TPA}} \beta_{\mathrm{DYE}}=1$. Figure 2 presents two equilibrium curves in the $\left(\Delta t_{L}, I_{0 L, D}\right)$-plane. The solid curve belongs to the parameters of Fig. 1 listed in Table $1 \quad\left(T_{0}=0.85\right.$, Gaussian pulse shape, $\left.\alpha^{(2)}=4 \times 10^{-12} \mathrm{~cm} / \mathrm{W}, \sigma_{\mathrm{ex}}=0\right)$. The curve indicates a minimum steady state pulse duration of 0.38 ps at $I_{0 L, D} \simeq 5 \times 10^{9} \mathrm{~W} / \mathrm{cm}^{2}$. It divides the plane into the two regions $I$ and $I I$. Transient pulse durations in region $I$ are shortened until the lower branch of the solid curve is reached. Initial pulses in region $I I$ are elongated. In region IIa the temporal broadening stops when the solid curve is reached (stable equilibrium). In region $I I b$ the pulses continuously broaden. The upper branch of the solid curve represents an unstable equilibrium.

Saturable absorbers with excited state absorption have a slightly reduced pulse shortening effect. For $\sigma_{\mathrm{ex}}=1 \times 10^{-16} \mathrm{~cm}^{2}$ and all other parameters the same as for the presented solid curve the minimum possible pulse duration becomes $\Delta t_{L, \min } \simeq 0.47 \mathrm{ps}$ at $I_{0 L, D} \simeq 4 \times 10^{9} \mathrm{~W} / \mathrm{cm}^{2}$. The use of higher concentrated saturable dyes shifts the equilibrium curve to lower pulse durations. A small signal dye transmission of $T_{0}=0.70 \quad\left(\sigma_{\mathrm{ex}}=0\right) \quad$ leads to $\Delta t_{L, \min } \simeq 0.23 \mathrm{ps}$ at $I_{0 L, D} \simeq 7 \times 10^{9} \mathrm{~W} / \mathrm{cm}^{2}$. Calculating the equilibrium 


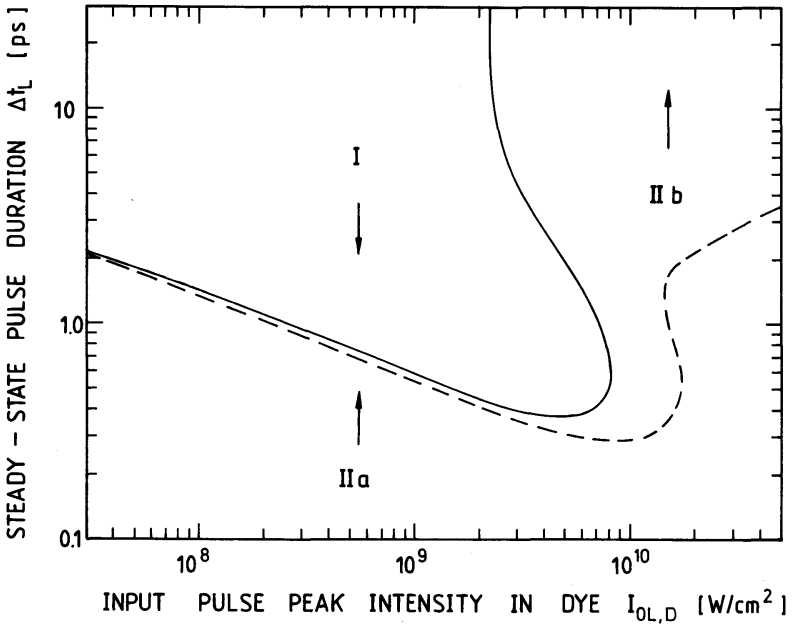

Fig. 2. Stationary equilibrium pulse duration versus pulse intensity. Solid curve represents data of Table 1 . The dashed curve is calculated for $\alpha^{(2)}=0$. The other parameters are the same as for the solid curve. Pulses in region $I$ are shortened, in region II are broadened

curve for the saturable absorber No. 5 (from Kodak or Lambda Physik) with the parameters $\sigma_{D}=3 \times 10^{-16}$ $\mathrm{cm}^{2}, \tau_{D}=2.7 \mathrm{ps}$ [16] and $\sigma_{\mathrm{ex}}=0$ (assumed) leads to practically the same result as for the dye Kodak No. 9860. Despite the negligible differences in the stationary equilibrium behaviour, the transient pulse development in real pulsed mode-locked lasers is different for mode-locking dye No. 5 and No. 9860 $[17,18]$.

The dashed curve in Fig. 2 is calculated without twophoton absorption. The other parameters are the same as for the solid curve (Table 1). A minimum stationary pulse duration of $\Delta t_{L, \min } \simeq 0.3 \mathrm{ps}$ is achieved at $I_{0 L, D} \simeq 10^{10} \mathrm{~W} / \mathrm{cm}^{2}$. Initial pulses below the curve are elongated while initial pulses above the curve are shortened. For absorption recovery times $\tau_{D}$ longer than the stationary equilibrium duration $\Delta t_{L}\left(I_{O L, D}\right)$ the bleaching and pulse shortening becomes nearly independent of $\tau_{D}$ [19] and the stationary equilibrium curve $\Delta t_{L}\left(I_{O L, D}\right)$ (dashed curve) remains unchanged. In this case the bleaching behaviour is determined by the saturation energy $E_{S}=h v_{L} / \sigma_{D}$. Large absorption cross-sections shift the equilibrium pulse duration curve to lower intensity values.

\section{Transient Pulse Development}

The above discussion about the stationary pulse duration does not indicate how fast the equilibrium is approached. To study the transient behaviour we simulated the pulse development in the nonlinear laser region [9].
At the $j$ th round trip in the nonlinear phase the intensity distribution $I_{L, j}(t)$ changes to

$I_{L, j+1}\left(\beta_{\mathrm{GAIN}, j} t\right)=I_{L, j}(t) G_{j} R_{1} R_{2} T_{l} T_{D, j}(t) T_{\mathrm{TPA}, j}(t)$.

The temporal broadening due to the gain medium is taken into account by rescaling the time axis after each round-trip from $t_{j}=t$ to $t_{j+1}=\beta_{\mathrm{GAIN}, j} t$. The light amplification in the active medium is

$G_{j}=\frac{1+\left(G_{N}-1\right) \delta}{R_{1} R_{2} T_{l} T_{0}^{2}}$.

The net gain per round-trip at the end of the linear phase is set to $G_{N}=1.03$ in the presented calculations. The factor $\delta \leqq 1$ takes into account gain depletion due to reduction of inversion ( $\delta=0.98$ used in calculations). The double-pass pulse transmission through the contacted dye cell $T_{D}(t)=I_{L \text {, out }}(t) / I_{L \text {, in }}(t)$ (in linear region $T_{D}=T_{0}^{2}$ ) is calculated by solving (8) and (9) [9]. The two-photon transmission $T_{\mathrm{TPA}, j}(t)$ is given by (6).

The calculations start at the end of the linear phase with an initial pulse intensity

$I_{L, 1}(t)=I_{0 L, 1}\left[\exp \left(-4 \ln 2 t^{2} / \Delta t_{0}^{2}\right)(1-\beta)+\beta\right]$.

Equation (12) approximates the real intensity distribution by the most intense spike with peak intensity $I_{O L, 1}$ and a constant background level $I_{B}=\beta I_{0 L, 1}$. The background intensity $I_{B}$ is set equal to the mean spike intensity $\langle I\rangle$ in the resonator which is given by $\langle I\rangle=\beta I_{0 L, 1} \approx I_{0 L, 1} / \ln (2 m)$, where $m=T /\left(2 \Delta t_{0}\right)$ is the number of pulses within the resonator round-trip time $T=2 L / c$ ( $L$ resonator length, $L=1.5 \mathrm{~m}$ in our case) [6].

In Fig. 3 numerical results for a mode-locked Nd : glass laser with saturable dye Kodak No. 9860 are presented (parameters in Table 1). The initial pulse parameters are taken from theoretical studies of the prelaser and linear laser phase [20] and are experimentally verified by measuring the spectral width of the free-running laser (without saturable absorber). After a certain intensity level is reached the laser intensity is kept constant for further round-trips as is indicated in Fig. 3a. The calculated pulse durations versus the number of round-trips in the nonlinear phase are shown in Fig. 3b. For the constant intensity region the Curves 1-3 start in region $I$ of Fig. 2 and pulse shortening is achieved. About 200 round-trips are necessary to approach the steady state equilibrium. The constant intensity values of Curves 4 and 5 $\left(I_{0 L, D}=6.5 \times 10^{9} \mathrm{~W} / \mathrm{cm}^{2}\right.$ and $\left.2.6 \times 10^{10} \mathrm{~W} / \mathrm{cm}^{2}\right)$ lie in region $I I b$ and the pulses are gradually elongated. The steady state limits $1^{\prime}-3^{\prime}$ expected from Fig. 2 are not reached since the evolving temporal pulse profile deviates from Gaussian shape. 


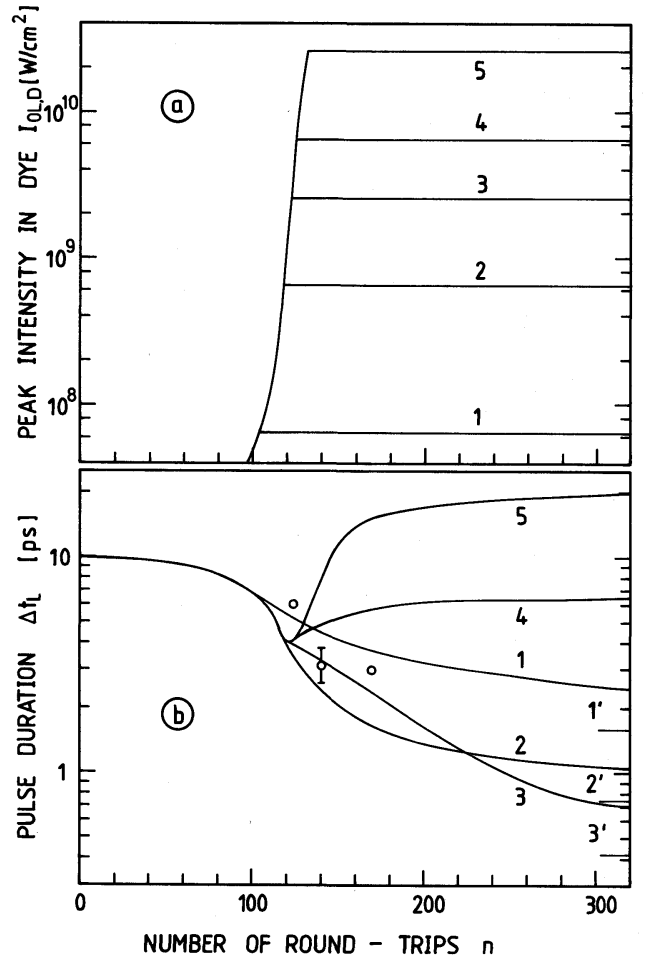

Fig. 3a and b. Pulse development in mode-locking region of the laser. (a) Development of the peak pulse intensity. (b) Corresponding pulse durations. Parameters of Table 1 are used in the calculations. Experimental points belong to pulses limited to $I_{0 L, D} \simeq 3 \times 10^{10} \mathrm{~W} / \mathrm{cm}^{2}$ with an intracavity Pockels cell. They should follow Curve 3 (stationary intensity $I_{0 L, D}=2.6 \times 10^{9}$ $\mathrm{W} / \mathrm{cm}^{2}$ )

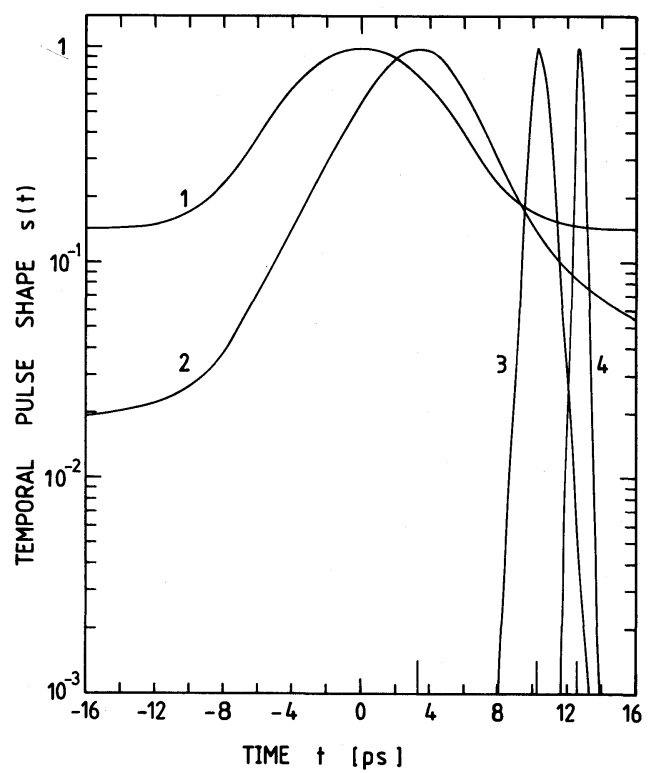

Fig. 4. Temporal pulse shape along the pulse train. Curve (1), shape at end of linear phase, (2) after 100, (3) 200, and (4) 300 round-trips in nonlinear phase. Curves belong to pulse development depicted by Curve 2 in Fig. 3 (stationary intensity $I_{0 L, D}=6.5 \times 10^{8} \mathrm{~W} / \mathrm{cm}^{2}$ ) and data of Table 1
The approached stationary pulse duration is independent of the initial pulse duration. In the transient mode-locking region of the laser, shorter starting pulses result in shorter final pulse durations $[9,21-24]$ if the net gain per round-trip is high enough that the initial pulse does not die out and only the trailing background signal survives. (Curves 2 and 2 in [9, Fig. 4] are correct for $G_{N} \simeq 1.05$. For $G_{N}=1.03$ the initial pulse is absorbed and the background signal at the trailing edge of the pulse forms a pulse with duration longer than Curves $l$ and $l^{\prime}$ of the same picture.)

Figure 4 shows the temporal pulse shape at the beginning of the mode-locking region, after 100, 200, and 300 round-trips for Curve 2 of Fig. 3. An asymmetric shape is formed due to the finite absorption recovery time and the background signal. The calculated pulse asymmetry is in agreement with experimental findings $[25,26]$.

\section{Prospects of Short Pulse Generation}

In conventional passively mode-locked lasers the optimum intensity region of efficient pulse shortening is passed too fast (Fig. 3a) and the minimum possible pulse duration is not achieved. The obtained peak intensity in the pulse train maximum can only be slightly influenced by mirror reflectivity. The region of pulse broadening by two-photon absorption is reached in mode-locked Nd: glass lasers. The intensity region of optimum pulse shortening in the absorber may be brought to the region of maximum peak pulse intensity by changing the resonator configuration and appropriate positioning the absorber cell in the resonator (influence of factor $\kappa=I_{0 L, D} / I_{0 L, A}$ ) or by applying a lens system in the resonator. Positioning the dye cell to a low intensity position in the resonator makes it difficult to start the mode-locking process since then even the most intense laser spike of the linear laser region does not reach the mode-locking threshold [20]. The use of two dye cells at different positions overcomes this problem as was checked experimentally.

Another possibility of pulse shortening is to fix the laser intensity in the resonator to the optimum pulse shortening region with an intensity limiter. In preliminary experiments we inserted a dielectric polarizer and a Pockels cell into the oscillator. With a photodiode triggered krytron system the Pockels cell was operated [27] when the laser intensity approached a value of $I_{0 L, D}$ around $3 \times 10^{9} \mathrm{~W} / \mathrm{cm}^{2}$. The voltage to the Pockels cell was regulated in such a way that the gain per round-trip became equal to the loss at the polarizer. The circulating pulse intensity remained 
approximately constant over up to fifty round-trips. The intensity limitation by the Pockels cell and polarizer does not cause a pulse broadening as intensity limitation by two-photon absorption does [28]. The pulse duration could be shortened from 6 to 3 ps. The experimental points are included in Fig. 3. They should follow the theoretical Curve 3 which was calculated for $I_{O L, D}=2.6 \times 10^{9} \mathrm{~W} / \mathrm{cm}^{2}$. The agreement between the experimental points and the calculated curve is fairly good.

\section{Conclusions}

The temporal pulse development in passively modelocked lasers was studied. The numerical examples were restricted to a pulsed passively mode-locked $\mathrm{Nd}$ : glass lasers but the theoretical considerations apply as well to other passively mode-locked laser systems. Without external manipulations the optimum intensity region for efficient pulse shortening is passed too fast and high intensity values are approached, where nonlinear loss mechanisms broaden the pulse duration. The insertion of a Pockels cell intensity limiter opens the possibility of optimum pulse shortening in the laser oscillator.

Acknowledgements. The authors are indepted to Prof. W. Kaiser for valuable discussions. They thank Th. Ascherl for technical assistance, P. Sperber for some investigations of lasers with two dye cells, and the Rechenzentrum of this University for disposal of computer time.

\section{References}

1. A.J. DeMaria, W.H. Glenn, M.J. Brienza, M.E. Mack: Proc. IEEE 57, 2 (1969)

2. P.G. Kryukov, V.S. Letokhov: IEEE J. QE-8, 766 (1972)
3. D. von der Linde: Appl. Phys. 2, 281 (1973)

4. A. Laubereau, W. Kaiser: Opto-Electronics 6, 1 (1974)

5. D.J. Bradley: In Ultrashort Light Pulses, ed. by S.L. Shapiro, Topics Appl. Phys. 18 (Springer, Berlin, Heidelberg, New York 1977) p. 17

6. W.H. Lowdermilk: In Laser Handbook, Vol. III, ed. by M.L. Stitch (North-Holland, Amsterdam 1979) p. 361

7. M.S. Demokan, Mode-Locking: In Solid-State and Semiconductor Lasers (Research Studies Press, Chichester 1982)

8. A. Penzkofer, W. Kaiser: Appl. Phys. Lett. 21, 547 (1972)

9. F. Graf, C. Löw, A. Penzkofer: Opt. Commun. 47, 329 (1983)

10. J. Herrman, F. Weidner, B. Wilhelmi: Appl. Phys. B 26, 197 (1981)

11. A. Penzkofer: Opto-Electronics 6, 87 (1974)

12. A. Penzkofer, W. Blau: Opt. Quantum Electron. 15, 325 (1983)

13. W. Blau, R. Reber, A. Penzkofer: Opt. Commun. 43, 210 (1983)

14. D. Kühlke, W. Rudolph, B. Wilhelmi: IEEE J. QE-19, 526 (1983)

15. D. Kühlke, W. Rudolph: Opt. Quantum Electron 16, 57 (1984)

16. B. Kopainsky, W. Kaiser, K.H. Drexhage: Opt. Commun. 32, $451(1980)$

17. C. Kolmeder, W. Zinth: Appl. Phys. 24, 341 (1981)

18. R.R. Alfano, N.H. Schiller, G.A. Reynolds: IEEE J. QE-17, 290 (1981)

19. G. Grönninger, A. Penzkofer: Opt. Quantum Electron. (1984, to be published)

20. A. Penzkofer: To be published

21. M.W. McGeoch: Opt. Commun. 7, 116 (1973)

22. D. von der Linde, K.F. Rodgers: Opt. Commun. 8, 91 (1973)

23. E.M. Gordeev, P.G. Kryukov, Yu.A. Matveets, B.M. Stepanov, S.D. Fachenko, S.V. Chekalin, A.V. Sharkov: Sov. J. Quantum Electron. 5, 129 (1975)

24. H. Graener, A. Laubereau: Opt. Commun. 37, 138 (1981)

25. J. Wiedmann, A. Penzkofer: Opt. Commun. 30, 107 (1979)

26. W. Leupacher, A. Penzkofer: Appl. Phys. B 29, 263 (1982)

27. J. Biebl, A. Penzkofer: J. Phys. E 13, 1328 (1980)

28. J.M. Ralston, R.K. Chang: Appl. Phys. Lett. 15, 164 (1969) 